\title{
Circular Polarization Observations and Magnetic Fields of $O$ Stars
}

\author{
Natallia P. Sudnik and Alexander F. Kholtygin \\ Saint-Petersburg State University, 28 Universitetskiy av., Saint-Petersburg, 198504, Russia
}

\begin{abstract}
The results of a search for line profile variability (LPV) and polarimetric line profile variability (pLPV) in spectra of $\mathrm{O}$ stars are reported. The observations were made with the $1.8-\mathrm{m}$ telescope of the Korean Bohyunsan Optical Astronomical Observatory (BOAO) and the 1-m and 6$\mathrm{m}$ telescopes of the Special Astrophysical Observatory (SAO), Russia. Regular LPV in the spectra of all program stars are found. We analyze the connection between the presence of magnetic field and nitrogen abundance anomalies, and the influence of weak magnetic fields on pLPV. The statistical properties of mean magnetic fields and magnetic fluxes of OB stars are studied.
\end{abstract}

Keywords: line: profiles — polarization — stars: oscillations — stars: early-type — stars: magnetic fields

PACS: 97.20.Ec, 97.10.Ld, 97.30.Eh, 97.10.Sj

\section{OBSERVATIONS}

The observations we analyzed were made in 1997-2010 at SAO and at BOAO. More than 1000 spectra of 15 OB stars ( $\xi$ Per, $\varepsilon$ Per, $\alpha$ Cam, HD 35502, $\delta$ Ori, $\lambda$ Ori A, $\theta^{1}$ Ori C, $\zeta$ Ori A, 15 Mon, $\rho$ Leo, HD 93521, 85 Her, 19 Cep, $\lambda$ Cep and 10 Lac) were obtained. The 6-m telescope observations were made with the Lynx spectrograph (spectral resolution $R=60000$ ) and CCD $512 \times 512$ pixels, with the NES spectrograph $(R=60000)$ and CCD $1024 \times 1024$ and with the MSS spectrograph $(R=15000)$ and CCD $2 \mathrm{k} \times 2 \mathrm{k}$, while $1-\mathrm{m}$ telescope observations were made with the CEGS spectrograph $(R=45000)$ and CCD $1242 \times 1152$. The reduction of SAO spectra was made with the MIDAS package. Most of these stars were also observed in BOAO by using the $1.8-\mathrm{m}$ telescope equipped with the BOES spectrograph $(R=45000)$ and large CCD (2048 $x 4096$ pixels). The preliminary reduction of the CCD frames was done with IRAF.

\section{POLARIMETRIC LINE PROFILE VARIABILITY}

We study LPV in integral (Stokes parameter $I)$, left $\left(I_{L}\right)$ and right $\left(I_{R}\right)$ polarized components of the line profile in spectra of stars $\lambda$ Cep, $\zeta$ Ori A and $\lambda$ Ori A. We find that the patterns of the pLPV for left and right polarized components can differ.

For the detection of micro LPV of various nature we used the smooth Time Variation Spectra (smTVS) technique introduced by Kholtygin et al. (2006). The smTVS is 

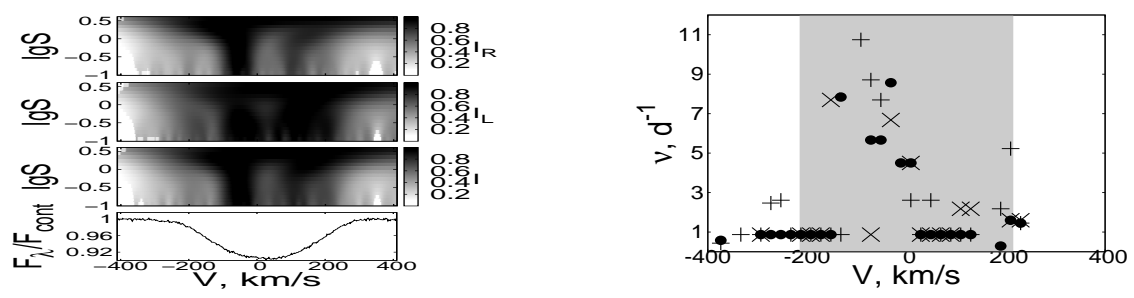

FIGURE 1. Left panel: Density plot diagram of smTVS for the $I_{R}, I_{L}$ and $I$ components of line profile and the mean profile for the line He I 4471 in spectra of the star $\lambda$ Cep (from top to bottom). Right panel: Fourier power spectra of the He I 4471 line in spectra of $\lambda$ Cep. Fourier components for $I$ are marked by circles, for $I_{L}$ by pluses and for $I_{R}$ by crosses. Each symbol corresponds to the component of the Fourier spectrum detected at significance level (FAP) $\alpha=10^{-3}$. The V $\sin i$ bar is highlighted by light-gray color.

described by:

$$
\operatorname{smTVS}(\lambda, S)=\frac{1}{N-1}\left(\sum_{i=1}^{n} g_{i}\left[I_{i}(\lambda, S)-\overline{I(\lambda, S)}\right]^{2} / \sum_{i=1}^{n} g_{i}\right),
$$

where $N$ is a number of spectra, $I_{i}(\lambda, S)$ is the line intensity in the spectrum with number $i$ at wavelength $\lambda$ smoothed with the Gaussian filter ( $S$ is a filter width) and normalized to the continuum level, $\overline{I(\lambda, S)}$ is the mean intensity at wavelength $\lambda$ averaged over all smoothed line profiles, $g_{i}$ is the relative weight of the $i^{\text {th }}$ observation. For $S=0$ the value of $\operatorname{smTVS}(\lambda, 0)$ corresponds to the TVS value introduced by Fullerton et al. [2].

Applying equation (1) to the left polarized components $I_{L}^{i}$ of the line we obtain the $\operatorname{smTVS}_{L}$ spectra, and we obtain the $\operatorname{smTVS}_{R}$ spectra in the case of using the right polarized components $I_{R}^{i}$. The density plot of the smTVS for the He I 4471 line in spectra of the star $\lambda$ Cep is shown in the Fig. 1. Darker areas correspond to higher amplitudes of the smTVS. The differences between $\operatorname{smTVS}_{L}$ and $\operatorname{smTVS}_{R}$ spectra are clearly seen.

To search for periodic profile variations, we carried out a Fourier analysis. False peaks were removed by a CLEAN procedure [3], modified by Vityazev [4]. Fig. 1 presents the Fourier power spectra of the time series for He I 4471 line profiles in spectra of $\lambda$ Cep for $I, I_{L}$ and $I_{R}$ line-profile components. We plot only those components of the Fourier spectra which exceed the significance level $\alpha=10^{-3}$ under the hypothesis that tehere is a strong peak in the white noise periodogram. We can see that there are regular components which can be detected in the Fourier spectrum for the $I_{L}$ component but cannot be detected for the $I_{R}$ component and vice versa.

\section{STATISTICS OF MAGNETIC FIELDS AND FLUXES OF O STARS}

Line profiles in spectra of O stars are usually strongly variable. The regular LPV can be connected with the presence of weak magnetic field on the stellar surface. At present the detection of a magnetic field was reported in literature for $20 \mathrm{O}$ stars which needs to be confirmed.

To investigate whether magnetic fields in massive $\mathrm{O}$ stars are ubiquitous or magnetic $\mathrm{O}$ stars form a special class of $\mathrm{O}$ stars with specific properties we use a statistical 
approach. As a characteristic of the mean stellar magnetic field we use the rms magnetic field $\langle B\rangle$ weakly depending on the geometry of the stellar magnetic field and from the time when the field was measured [5].

$$
\langle B\rangle=\sqrt{\frac{1}{n} \sum_{i=1}^{n}\left(B_{l}^{i}\right)^{2}},
$$

where $B_{l}^{i}$ is the mean longitudinal magnetic field for the measurement number $i$. As a measure of the reality of measured field value we use the next criteria:

$$
\langle B\rangle>2 \sigma_{B} \text { and } \chi^{2} / n>1,
$$

where

$$
\sigma_{B}=\sqrt{\frac{1}{n} \sum_{i=1}^{n}\left(\sigma_{B_{l}}^{i}\right)^{2}} \quad \text { and } \quad \chi^{2} / n=\frac{1}{n} \sum_{i=1}^{n} \frac{\left(B_{l}^{i}\right)^{2}}{\left(\sigma_{B_{l}}^{i}\right)^{2}} .
$$

Here $\sigma_{B_{l}}^{i}$ is the $r m s$ error of the $i^{t h}$ field measurement. The normalized distribution of the rms magnetic field of $\mathrm{O}$ stars is given in Fig. 2.

Morel et al. [6] suggest that magnetic B stars have an overabundance of N. For magnetic $\mathrm{O}$ stars we found only the abundance of $\mathrm{N}$ for three stars. $\zeta$ Ori and $10 \mathrm{Lac}$ have nearly solar $\mathrm{N}$ abundance, while HD 108 have a nitrogen abundance 6 times higher than solar one. The correlation of nitrogen overabundance and magnetic field for $\mathrm{O}$ stars remains unclear.

In Fig. 3 the distribution of Vsin $i$ for O stars is presented. Based on our comparison we can conclude that there is no significant difference between magnetic and non magnetic O stars.

Two color diagram $(\mathrm{B}-\mathrm{V})_{0}-(\mathrm{U}-\mathrm{B})_{0}$ for $\mathrm{O}$ stars is shown in Fig. 3.We may preliminary conclude that for magnetic $\mathrm{O}$ stars $(\mathrm{B}-\mathrm{V})_{0}<-0.24^{\mathrm{m}}$, but it can be this can be due to selection effects.

To estimate the magnetic fluxes for stars with known magnetic fields we use the following relation:

$$
F=\alpha 4 \pi\langle B\rangle R^{2}
$$

Here $R$ is the stellar radius, $\alpha$ is a constant depending on the geometry of the magnetic field. For a dipole field $\alpha \approx 5 / 3$, for more complex configurations $\alpha \approx 1$. We adopt $\alpha=1$
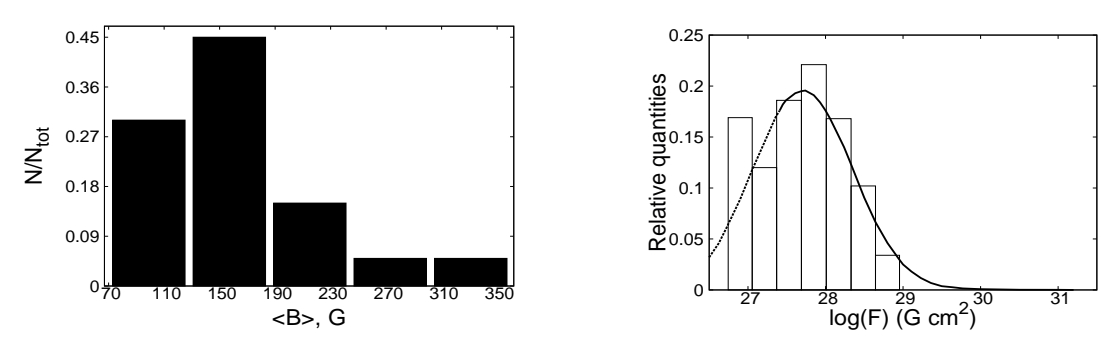

FIGURE 2. Left panel: Distribution of $\langle B\rangle$ for O stars. Right panel: Distribution of magnetic fluxes for massive OB stars. The solid line shows the approximation of the distribution by a log-normal law in the region $F>\bar{F}$. The dashed line is an extrapolation of this dependence for the lower values of $\mathrm{F}$. 

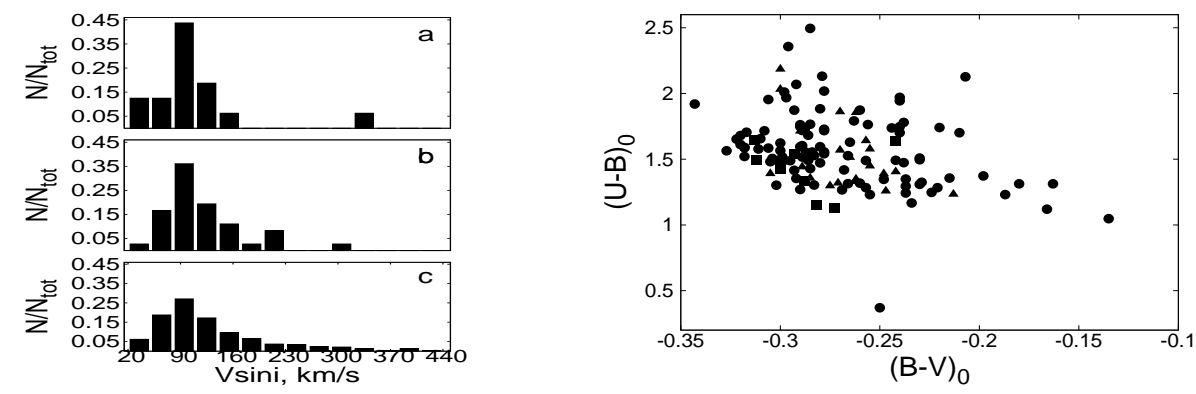

FIGURE 3. Left panel: Distribution of V $\sin i$ for magnetic (a), suspected magnetic (b) and remaining $\mathrm{O}$ stars (c). Right panel: Two color diagram $(\mathrm{B}-\mathrm{V})_{0}-(\mathrm{U}-\mathrm{B})_{0}$ for magnetic (squares), suspected magnetic (triangles) and remaining $\mathrm{O}$ stars (circles).

for all configurations. The distribution of magnetic fluxes for massive OB stars is given in Fig. 2. We can see that distribution of magnetic fluxes is asymmetric due the lack of stars with small magnetic fluxes. At the same time, the behavior of the distribution for large fluxes $F>\bar{F}$, where $\bar{F}$ is the mean flux for the considered group of stars is more regular. We suppose that in the region $F>\bar{F}$ the distribution of magnetic fluxes can be described by a log-normal law.

\section{CONCLUSIONS}

The following conclusions can be drawn from our analysis of pLPV and statistics of magnetic fields of $\mathrm{O}$ stars:

- All studied stars show micro LPV with an amplitude of about $0.5-3 \%$.

- It is possible that LPV for left and right polarized components of the line profiles can differ.

- The difference between magnetic and non-magnetic $\mathrm{O}$ stars remains unclear.

\section{ACKNOWLEDGMENTS}

We thank A. P. Igoshev for permission to use the distribution of magnetic fluxes he obtained for OB stars. This study was supported by the Project 6.38.73.2011 of the Saint-Petersburg State University (SPbSU). N. P. S. acknowledges SPbSU for support by grant 6.41 .364 .2011 .

\section{REFERENCES}

1. A. F. Kholtygin, T. E. Burlakova, S. N. Fabrika et al., Astronomy Reports, 50, 887-901 (2006).

2. A. W. Fullerton, D. R.Gies and C. T. Bolton, Astrophys. J. Suppl. Ser, 103, 475-512 (1996).

3. D. H. Roberts, J. Lehar and J. W. Dreher, Astrophys. J., 93, 4, 968-989 (1987).

4. V. V. Vityazev, Analysis of irregular time series, Publisher of SPbSU, 2001, pp. 1-69.

5. A. F. Kholtygin, S. N. Fabrika, N. A. Drake et al., Astronomy Letters, 36, 5, 370-379 (2010).

6. T. Morel, S. Hubrig and M. Briquet, Astron. and Astrophys, 481, 453-463 (2008). 